\title{
Consensus of Multiagent Systems with Sampled Information and Noisy Measurements
}

\author{
Zhao-Jun Tang, ${ }^{1,2}$ Ting-Zhu Huang, ${ }^{2}$ and Jin-Liang Shao ${ }^{2}$ \\ ${ }^{1}$ School of Science, Chongqing Jiaotong University, Chongqing 400074, China \\ ${ }^{2}$ School of Mathematical Sciences, University of Electronic Science and Technology of China, Chengdu 611731, China \\ Correspondence should be addressed to Ting-Zhu Huang; tingzhuhuang@126.com
}

Received 26 October 2012; Accepted 4 January 2013

Academic Editor: Vimal Singh

Copyright (c) 2013 Zhao-Jun Tang et al. This is an open access article distributed under the Creative Commons Attribution License, which permits unrestricted use, distribution, and reproduction in any medium, provided the original work is properly cited.

\begin{abstract}
We consider consensus problems of first-order multiagent systems with sampled information and noisy measurements. A distributed stochastic approximation type algorithm is employed to attenuate the measurement noises. We provide conditions under which almost sure strong consensus is guaranteed for fixed and switching directed network topologies. Simulation results are provided to illustrate the theoretical results.
\end{abstract}

\section{Introduction}

In recent years, distributed coordination control of multiagent systems has received compelling attention from the control community. As a critical issue for coordination control, consensus means that the group of agents reach an agreement on a common value via local communication. Consensus problems are closely related to many different problems that involve interconnection of dynamic systems in various fields of science and engineering, such as synchronization of coupled oscillators, flocking theory, fast consensus in small worlds, rendezvous in space, distributed sensor fusion in sensor networks, and distributed formation control (see [1]).

Vicsek et al. [2] provided a variety of simulation results which demonstrate that simple distributed algorithms allow all nodes to eventually reach an agreement. The work in [3] provided a theoretical explanation for the observed behavior of the Vicsek model. Up to now, a variety of consensus protocols have been developed to deal with dynamic topologies [4-6], time delays [7-9], finite-time convergence [10-12], and random network topologies $[13,14]$, just to name a few.

In many applications envisioned, due to the application of digital sensors and controllers, the information exchange among the group of agents may only occur at sampling instants. Thus, it is more practical to consider the consensus problems with sampled-data information. Moreover, with sampled-data control, many benefits can be achieved such as flexibility, robustness, and low cost. Some results about consensus problems for multi-agent systems via sampleddata control have been reported [15-18]. On the other hand, it was assumed that in most existing works each agent can obtain the accurate information from its neighbors. Obviously, this assumption is impractical for real communication channels since the information exchange is often corrupted by various noises. Consensus seeking with noisy measurements has attracted the attention of some researchers. Huang and Manton [19] studied the consensus problem of discretetime multi-agent systems with fixed and undirected topology under noisy measurements and proposed stochastic approximation-type protocols with a decreasing step size. Subsequently, they extended the results of [19] to the directed topology case. Li and Zhang [20] investigated the averageconsensus problem of discrete-time multi-agent systems with time-varying topologies and noisy measurements. Different from discrete-time systems in which the sampling period is often assumed to be 1 , the impact of the sampling period has to be considered for sampled-data control systems. In most existing results concerning consensus problems based on sampled-data control, it is shown that the sampling period must satisfy a certain condition to guarantee convergence. To 
the best of our knowledge, there are a few works considering the consensus problems with sampled information and noisy measurements. Li and Zhang [21] considered mean square average-consensus problems of multi-agent systems based on sampled-data control with noisy measurements and timeinvariant topology. Under the assumption that the interaction topology is undirected and connected, it was shown that the agents reach mean square average consensus if the sampling period is sufficiently small.

In this paper, we are interested in the consensus problems with sampled information and noisy measurements. That is, we assume that each agent can only obtain the noisy measurements of its neighbors' states at sampling instants. This paper is partly motivated by [21]. This paper makes the following contributions. First, for the case of fixed topology, only the existence of a spanning tree is required in this paper, which is much weaker than connected. Second, the case of timevarying topology is investigated, and we prove that the agents reach almost sure strong consensus provided each interaction topology is balanced and contains a spanning tree. Third, we prove that the convergence condition is independent of the sampling period due to the introduction of the step size. Nevertheless, it should be pointed out that both the step size and the sampling period have an impact on the convergence speed. Comparing with $[20,22]$, we study continuous-time multi-agent systems with sampled information instead of discrete-time systems. In addition, the method used in the convergence analysis in this paper is different from it in the aforementioned references.

The following notations will be used throughout this paper. Let $I$ be an identity matrix with appropriate dimension, and 1 be a column vector of all ones with appropriate dimension. For a given matrix $A, A^{T}$ denotes its transpose; $\|A\|$ denotes its 2-norm; let $\lambda_{\max }(A)$ and $\lambda_{\text {min }}(A)$ denote its maximum and minimum eigenvalues, respectively. A matrix $A$ is said to be positive stable if all of its eigenvalues have positive real parts. For a given random variable $\xi, E[\xi]$ denotes its mathematical expectation.

\section{Problem Formulation}

2.1. Graph Theory. Consider a multi-agent system consisting of $n$ agents labeled 1 through $n$. The interaction topology among the $n$ agents can be described by a digraph $\mathscr{G}=$ $(\mathscr{V}, \mathscr{E})$, where $\mathscr{V}=\{1, \ldots, n\}$ is the set of nodes and $\mathscr{E} \subseteq$ $\mathscr{V} \times \mathscr{V}$ is the set of edges of the graph. An edge of $\mathscr{G}$ is denoted by $(i, j)$, representing that agent $j$ can directly receive information from agent $i$. The set of neighbors of node $i$ is denoted by $\mathscr{N}_{i}=\{j \in \mathscr{V} \mid(j, i) \in \mathscr{E}\}$. A path in $\mathscr{G}$ is a sequence $i_{0}, i_{1}, \ldots, i_{m}$ of distinct nodes such that $\left(i_{j-1}, i_{j}\right) \in \mathscr{E}$ for $j=1, \ldots, m$. A directed graph $\mathscr{G}$ is strongly connected if there is a path between any two distinct nodes. A graph contains a spanning tree if there exists at least one node having a directed path to all other nodes.

The weighted adjacency matrix of the digraph $\mathscr{G}$ is denoted by $A=\left[a_{i j}\right] \in \mathbb{R}^{n \times n}$, where $a_{i j}>0$ if $(j, i) \in \mathscr{E}$ and $a_{i j}=0$ otherwise. The in-degree of node $i$ is defined as $\operatorname{deg}_{\text {in }}(i)=\sum_{j=1}^{n} a_{i j}$ and the out-degree is defined as $\operatorname{deg}_{\text {out }}(i)=$ $\sum_{j=1}^{n} a_{j i}$. Its degree matrix $D=\operatorname{diag}\left\{d_{1}, \ldots, d_{n}\right\}$ is a diagonal matrix, whose diagonal elements $d_{i}=\operatorname{deg}_{\text {in }}(i) . \mathscr{G}$ is called a balanced digraph, if $\operatorname{deg}_{\text {in }}(i)=\operatorname{deg}_{\text {out }}(i), i=1,2, \ldots, n$. The Laplacian matrix associated with the digraph $\mathscr{G}$ is defined as

$$
L_{\mathscr{G}}=D-A .
$$

Below is an important property of Laplacian $L_{\mathscr{G}}$.

Lemma 1 (see [6]). Zero is an eigenvalue of $L_{\mathscr{G}}$, and 1 is the associated right eigenvector. In addition, zero is a simple eigenvalue of $L_{g}$ and all the other eigenvalues have positive real parts if and only if the digraph $\mathscr{G}$ contains a spanning tree.

2.2. Consensus Protocols. Consider the following first-order integrator system of $n$ agents:

$$
\dot{x}_{i}(t)=u_{i}(t), \quad i=1, \ldots, n,
$$

where $x_{i} \in \mathbb{R}$ and $u_{i} \in \mathbb{R}$ are the state and control input of agent $i$, respectively.

In this paper, we assume that each agent can only obtain noisy measurements of the states of its neighbors at sampling instants. Denote the resulting measurement by agent $i$ of $x_{j}(k h)$ by

$$
\begin{array}{r}
y_{i j}(k h)=x_{j}(k h)+w_{i j}(k h), \quad j \in \mathcal{N}_{i}, \\
k \in \mathbb{Z}^{+}=\{0,1,2, \ldots\},
\end{array}
$$

where $h$ is the sampling period and $w_{i j}(k h)$ is the additive noise. The underlying probability space is denoted by $(\Omega, \mathscr{F}, P)$.

Definition 2 (see [19]). The agents are said to reach almost sure strong consensus if there exists a random variable $x^{*}$ such that $\lim _{t \rightarrow \infty} x_{i}(t)=x^{*}$ almost surely (a.s.), for all $i \in \mathscr{V}$.

Remark 3. When there is no noisy measurements, Definition 2 is equivalent to the definition of consensus for deterministic systems. That is, the agents are said to reach consensus if $\lim _{t \rightarrow \infty}\left(x_{i}(t)-x_{j}(t)\right)=0$ for any $i \neq j$.

Our objective is to design a distributed protocol so that the $n$ agents can reach almost sure strong consensus. For this end, we study the following distributed protocol [21]:

$$
\begin{aligned}
& u_{i}(t)=a(k) \sum_{j \in \mathcal{N}_{i}(k h)} a_{i j}\left(y_{i j}(k h)-x_{i}(k h)\right), \\
& \forall t \in[k h,(k+1) h), \quad k \in \mathbb{Z}^{+}, \quad i=1, \ldots, n,
\end{aligned}
$$

where $a(k)>0$ is the step size. Here, the introduction of the step size is to attenuate the noises, which is often used in classical stochastic approximation theory [23]. We introduce the following assumptions on the step size sequence:

$$
\begin{aligned}
& (\mathrm{A} 1) \sum_{k=0}^{\infty} a(k)=\infty, \sum_{k=0}^{\infty} a^{2}(k)<\infty, \\
& \left(\mathrm{Al}^{\prime}\right) \sum_{k=0}^{\infty} a(k)=\infty, \lim _{k \rightarrow \infty} a(k)=0 .
\end{aligned}
$$

Remark 4. Assumption $\left(\mathrm{Al}^{\prime}\right)$ is weaker than assumption (A1). The above assumptions imply that the impact of the 
noise can be attenuated as time goes on since $a(k) \rightarrow 0$ as $k \rightarrow \infty . \sum_{k=0}^{\infty} a(k)=\infty$ implies that $a(k)$ cannot decrease too fast since, otherwise, the agents may prematurely converge to different individual limits.

Write $x=\left(x_{1}, \ldots, x_{n}\right)^{T}$. The discrete-time model with zero-order hold of (2) is

$$
x_{i}((k+1) h)=x_{i}(k h)+h u_{i}(k h), \quad k \in \mathbb{Z}^{+} .
$$

With the protocol (4), (5) can be written in matrix form as

$$
x((k+1) h)=\left(I-a(k) h L_{\mathscr{G}(k h)}\right) x(k h)+a(k) h w(k h),
$$

where $w(k h)=\left(w_{1}(k h), \ldots, w_{n}(k h)\right)^{T}$ with $w_{i}(k h)=$ $\sum_{j=1}^{n} a_{i j} w_{i j}(k h)$. Denote $w(k h)=\left(w_{1}^{T}(k h), \ldots, w_{n}^{T}(k h)\right)^{T} \in$ $\mathbb{R}^{n^{2}}$ with $w_{i}(k h)=\left(w_{i 1}(k h), \ldots, w_{i n}(k h)\right)^{T} \in \mathbb{R}^{n}$. We introduce the following assumptions on the measurement noises.

(A2) $\left\{w(k h) \in \mathbb{R}^{n^{2}}, \mathscr{F}^{w}(k h)\right\}$ is a martingale difference sequence, $\sup _{k \geq 0} E\|w(k h)\|^{2}<\infty$, where and whereafter $\mathscr{F}^{w}(k h)$ denotes the $\sigma$-algebras $\sigma\{w(0), \ldots, w(k h)\}$ for $k \geq 0$.

Note that (A2) contains that $\left\{w_{i j}(k h), k \in \mathbb{Z}^{+}, i, j=\right.$ $1,2, \ldots, n,(j, i) \in \mathscr{E}\}$ are independent sequences with zero mean and uniformly bounded second-order moments as a special case.

\section{Main Results}

3.1. Fixed Topology. In this subsection, we consider the case of fixed topology. We begin by studying the noise-free case; that is, $w_{i j}=0, i, j=1, \ldots, n$. Then protocol (4) becomes

$$
\begin{array}{r}
u_{i}(t)=a(k) \sum_{j=1}^{n} a_{i j}\left(x_{j}(k h)-x_{i}(k h)\right), \\
\forall t \in[k h,(k+1) h), \quad i=1, \ldots, n,
\end{array}
$$

and system (6) becomes

$$
x((k+1) h)=(I-a(k) h L) x(k h) .
$$

We make the following assumption on the network topology.

(A3) $\mathscr{G}$ has a spanning tree.

It is not easy to analyze the convergence of system (8) directly since $L$ is not positive stable. To deal with this problem, we need the following lemma.

Lemma 5 (see [22]). Assuming (A3), denote $\mathscr{C}(L)=\{\phi \in$ $\left.R^{n \times(n-1)} \mid \operatorname{span}\{\phi\}=\operatorname{span}\{L\}\right\}$. For any given $Q_{1} \in \mathscr{C}(L)$, the matrix $Q=\left(1, Q_{1}\right)$ is nonsingular and

$$
Q^{-1} L Q=\left(\begin{array}{ll}
0 & \\
& H
\end{array}\right)
$$

where $H \in \mathbb{R}^{(n-1) \times(n-1)}$ is positive stable. In addition, let $Q^{-1}=$ $\left(\begin{array}{c}q^{T} \\ Q_{2}\end{array}\right)$, where $q \in \mathbb{R}^{n}$; then $q^{T} L=0$ and $q^{T} \mathbf{1}=1$.
Following the notation in Lemma 5, let $\delta(k h)=$ $\left(\delta_{1}(k h), \ldots, \delta_{n}(k h)\right)^{T}=Q^{-1} x(k h)$. Then we can obtain the following system associated with system (8):

$$
\widehat{\delta}((k+1) h)=(I-a(k) h H) \widehat{\delta}(k h),
$$

where $\widehat{\delta}(k h)=\left(\delta_{2}(k h), \ldots, \delta_{n}(k h)\right)^{T}$. Furthermore, we have the following relationship:

$$
\begin{gathered}
x(k h)=\delta_{1}(k h) \mathbf{1}+Q_{1} \widehat{\delta}(k h), \\
\delta_{1}((k+1) h)=\delta_{1}(k h)+a(k) h q^{T} w(k h) .
\end{gathered}
$$

Noticing that $H$ is positive stable, by Lyapunov theorem [24], there exists a positive definite matrix $P$ such that

$$
P H+H^{T} P=I \text {. }
$$

Before moving on, we need the following lemma.

Lemma 6 (see [25]). Let $\{u(k), k=0,1, \ldots\},\{\alpha(k), k=$ $0,1, \ldots\}$, and $\{q(k), k=0,1, \ldots\}$ be real sequence, satisfying $0<q(k) \leq 1, \alpha(k) \geq 0, k=0,1, \ldots, \sum_{k=0}^{\infty} q(k)=\infty$, $\alpha(k) / q(k) \rightarrow 0, k \rightarrow \infty$, and

$$
u(k+1) \leq(1-q(k)) u(k)+\alpha(k) .
$$

Then $\lim \sup _{k \rightarrow \infty} u(k) \leq 0$. In particular, if $u(k) \geq 0, k=$ $0,1, \ldots$, then $u(k) \rightarrow 0$ as $k \rightarrow \infty$.

Theorem 7. Apply protocol (7) to system (2). Assume that $\left(A 1^{\prime}\right)$ and (A2) hold; then the $n$ agents reach consensus.

Proof. Take a Lyapunov function

$$
V(k)=\widehat{\delta}^{T}(k h) P \widehat{\delta}(k h),
$$

where $P$ is defined in (13). Then,

$$
\begin{aligned}
V(k+1)=V(k)-a(k) h \widehat{\delta}^{T}(k h) \widehat{\delta}(k h) \\
+a^{2}(k) h^{2} \widehat{\delta}^{T}(k h) H^{T} H \widehat{\delta}(k h) .
\end{aligned}
$$

Using the fact that

$$
\begin{aligned}
\lambda_{\min }(P) \widehat{\delta}^{T}(k h) \widehat{\delta}(k h) & \leq \widehat{\delta}^{T}(k h) P \widehat{\delta}(k h) \\
& \leq \lambda_{\max }(P) \widehat{\delta}^{T}(k h) \widehat{\delta}(k h)
\end{aligned}
$$

it follows that

$$
\begin{aligned}
& V(k+1) \\
& \quad \leq\left[1-a(k) h \frac{1}{\lambda_{\max }(P)}+a^{2}(k) h^{2} \frac{\lambda_{\max }\left(H^{T} H\right)}{\lambda_{\min }(P)}\right] V(k) .
\end{aligned}
$$

By $\left(\mathrm{Al}^{\prime}\right)$, there exists a $k_{0}>0$ such that $a(k) \leq$ $\lambda_{\text {min }}(P) / 2 h \lambda_{\max }\left(H^{T} H\right) \lambda_{\max }(P)$ and $a(k) \leq \lambda_{\min }(P) / h$ for all $k \geq k_{0}$. Thus,

$$
V(k+1) \leq\left[1-\frac{1}{2} a(k) h \frac{1}{\lambda_{\max }(P)}\right] V(k), \quad k \geq k_{0} .
$$


In light of Lemma 6 , we have $\lim _{k \rightarrow \infty} V(k)=0$ by noting that $\sum_{k=0}^{\infty} a(k)=\infty$. This implies $\lim _{k \rightarrow \infty} \widehat{\delta}(k h)=0$. Therefore, the conclusion readily follows from (11).

Now we investigate the case of having noisy measurements. We can obtain the following system associated with system (6):

$$
\widehat{\delta}((k+1) h)=(I-a(k) h H) \widehat{\delta}(k h)+a(k) h Q_{2} w(k h),
$$

where $H$ and $Q_{2}$ are defined in Lemma 5.

Theorem 8. Consider system (20). Assume that (A1)-(A3) hold; then

$$
\lim _{k \rightarrow \infty}\|\widehat{\delta}(k h)\|^{2}=0 \quad \text { a.s. }
$$

Proof. Choose a Lyapunov function

$$
V(k)=\widehat{\delta}^{T}(k h) P \widehat{\delta}(k h),
$$

where $P$ is defined in (13). By a similar argument to that in the proof of (18), we can obtain

$$
\begin{aligned}
& V(k+1) \\
& \leq {\left[1-a(k) h \frac{1}{\lambda_{\max }(P)}+a^{2}(k) h^{2} \frac{\lambda_{\max }\left(H^{T} H\right)}{\lambda_{\min }(P)}\right] V(k) } \\
&+2 a(k) h \hat{\delta}^{T}(k h) \times\left(I-a(k) h H^{T}\right) P Q_{2} w(k h) \\
&+a^{2}(k) h^{2} w^{T}(k h) Q_{2}^{T} Q_{2} w(k h) .
\end{aligned}
$$

Taking the expectation of the above, given $\{V(s): s \leq k\}$, yields

$$
\begin{aligned}
& E\{V(k+1) \mid V(s): s \leq k\} \\
& \leq {\left[1-a(k) h \frac{1}{\lambda_{\max }(P)}+a^{2}(k) h^{2} \frac{\lambda_{\max }\left(H^{T} H\right)}{\lambda_{\min }(P)}\right] V(k) } \\
&+C_{1} a^{2}(k),
\end{aligned}
$$

for some constant $C_{1}>0$, where we have used the fact that $E\left[\widehat{\delta}^{T}(k h)\left(I-a(k) h H^{T}\right) P Q_{2} w(k h)\right]=0$ by noting that $\widehat{\delta}(k h) \epsilon$ $\mathscr{F}^{w}((k-1) h)$.

By a similar argument to that in the proof of (19), there exists a $k_{0}>0$ such that

$$
\begin{aligned}
E\{V(k+1) \mid V(s): s \leq k\} \leq & {\left[1-\frac{1}{2} a(k) h \frac{1}{\lambda_{\max }(P)}\right] } \\
& \times V(k)+C_{1} a^{2}(k), \quad \forall k \geq k_{0} .
\end{aligned}
$$

We will make use of the following lemma to finish up our proof.
Lemma 9 (see [25]). Consider a sequence of nonnegative random variables $\{V(k)\}_{k \geq 0}$ with $E\{V(0)\}<\infty$. Let

$$
\begin{aligned}
& E\{V(k+1) \mid V(k), \ldots, V(1), V(0)\} \\
& \quad \leq\left(1-c_{1}(k)\right) V(k)+c_{2}(k),
\end{aligned}
$$

where

$$
\begin{gathered}
0 \leq c_{1}(k) \leq 1, \quad c_{2}(k) \geq 0, \quad \forall k, \\
\sum_{k=0}^{\infty} c_{2}(k)<\infty, \quad \sum_{k=0}^{\infty} c_{1}(k)=\infty, \\
\lim _{k \rightarrow \infty} \frac{c_{1}(k)}{c_{2}(k)}=0 .
\end{gathered}
$$

Then, $V(k)$ a.s. converges to zero; that is,

$$
\lim _{k \rightarrow \infty} V(k)=0 \quad \text { a.s. }
$$

It is not hard to show that conditions (26) and (27) are satisfied. Therefore, $\lim _{k \rightarrow \infty} V(k)=0$, a.s., which implies the conclusion.

Let $J=(1 / n) 1 \mathbf{1}^{T}$. The following lemma will be used to obtain the main result of this subsection.

Lemma 10 (see [26]). Let $V(k)=\|x(k h)-J x(k h)\|^{2}$. Then

$$
\lim _{k \rightarrow \infty} V(k)=0 \quad \text { a.s. }
$$

if and only if

$$
\lim _{k \rightarrow \infty} x(k h)=c 1 \quad \text { a.s., }
$$

for some $c \in \mathbb{R}$.

Theorem 11. Apply protocol (4) to system (2). Assume that (A1)-(A3) hold; then the $n$ agents reach almost sure strong consensus.

Proof. From (11), we have

$$
\begin{aligned}
\|(I-J) x(k h)\|^{2} & =\left\|(I-J) Q_{1} \widehat{\delta}(k h)\right\|^{2} \\
& \leq\left\|Q_{1}^{T}(I-J) Q_{1}\right\|\|\widehat{\delta}(k h)\|^{2},
\end{aligned}
$$

by noting that $(I-J) \mathbf{1}=0$ and $(I-J)^{2}=I-J$. By Theorem 8 , we have

$$
\lim _{k \rightarrow \infty}\|x(k h)-J x(k h)\|^{2}=0 \quad \text { a.s. }
$$

Invoking Lemma 10, we have

$$
\lim _{k \rightarrow \infty} x(k h)=c 1 \text { a.s., }
$$

for some constant $c$. 


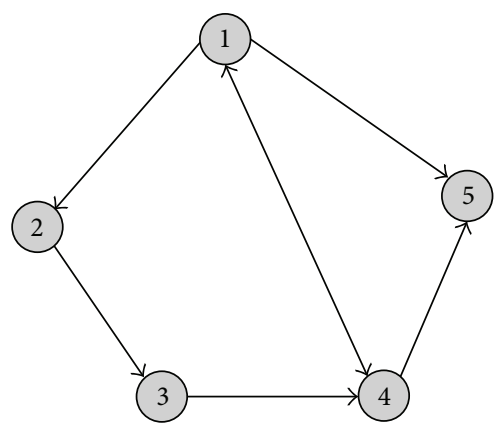

FIGURE 1: Interaction topology $\mathscr{G}_{1}$.

At the same time, since $\sum_{k=0}^{\infty} a^{2}(k) E\|w(k h)\|^{2}<\infty$ by (A2), it follows that

$$
\sum_{k=0}^{\infty} a^{2}(k) E\|w(k h)\|^{2}<\infty .
$$

By the martingale convergence theorem [27], it follows that $\sum_{k=0}^{\infty} a(k) w(k h)$ converge a.s., as $k \rightarrow \infty$. Then, we have

$$
\lim _{k \rightarrow \infty} a(k) w(k h)=0 \quad \text { a.s. }
$$

Notice that

$$
\begin{array}{r}
x(t)=[I-a(k)(t-k h) L] x(k h)+a(k) h w(k h), \\
\forall t \in[k h,(k+1) h) .
\end{array}
$$

This together with (33) and (35) leads to

$$
\lim _{t \rightarrow \infty} x(t)=c 1 \quad \text { a.s., }
$$

for some constant $c$, which completes the proof.

Remark 12. In most existing works concerning consensus problems based on sampled-data control, there is a requirement on the sampling period $h$ to guarantee convergence since there is no step size or step size $a(k) \equiv 1$. For instance, when $a(k) \equiv 1$ we can easily show that the sampling period must satisfy $h \leq 1 / \lambda_{\max }(L)$ to guarantee the convergence of system (8). However, by Theorems 7 and 11, we find that the convergence condition is independent of the sampling period $h$ for both noise-free and noisy measurements cases. This is due to the introduction of the step size. In other words, the introduction of the step size is necessary for enhancing the robustness of the sampled-data consensus protocol against sampling period.

3.2. Time-Varying Topology. In this subsection, we consider the case when the network topology changes dynamically. In order to describe the time-varying topology, we define a switching signal $\sigma(k): \mathbb{Z}^{+} \rightarrow \mathscr{P}=\{1,2, \ldots, N\}$, where $N$ is the total number of all possible graphs describing the interaction topologies. At each sampling time $k h$, the underlying graph is denoted by $\mathscr{G}_{\sigma(k)}$. Then, we rewrite system (6) as

$$
x((k+1) h)=\left(I-a(k) h L_{\sigma(k)}\right) x(k h)+a(k) h w(k h),
$$

where $L_{\sigma(k)}$ denotes the Laplacian matrix associated with the diagraph $\mathscr{G}_{\sigma(k)}$.

We introduce the following assumption on the network topology.

(A4) $\mathscr{G}_{\sigma(k)}$ is balanced for any $k \in \mathbb{Z}^{+}$.

Under (A4), there exists an orthogonal matrix $U=$ $\left((1 / \sqrt{n}) \mathbf{1}, U_{1}\right) \in \mathbb{R}^{n \times n}$ such that

$$
U^{T} L_{\sigma(k)} U=\left(\begin{array}{ll}
0 & \\
& H_{\sigma(k)}
\end{array}\right), \quad \forall k \in \mathbb{Z}^{+},
$$

where $H_{\sigma(k)} \in \mathbb{R}^{(n-1) \times(n-1)}$.

Let $\delta(k h)=\left(\delta_{1}(k h), \ldots, \delta_{n}(k h)\right)^{T}=U^{T} x(k h)$. We can obtain the following system associated with system (38):

$$
\widehat{\delta}((k+1) h)=\left(I-a(k) h H_{\sigma(k)}\right) \widehat{\delta}(k h)+a(k) h U_{1}^{T} w(k h),
$$

where $\widehat{\delta}(k h)=\left(\delta_{2}(k h), \ldots, \delta_{n}(k h)\right)^{T}$. In addition, we have

$$
x(k h)=\frac{1}{\sqrt{n}} \delta_{1}(k h) \mathbf{1}+U_{1} \widehat{\delta}(k h) .
$$

Theorem 13. Consider system (40). Suppose that (A1)-(A4) hold; then

$$
\lim _{k \rightarrow \infty}\|\widehat{\delta}(k h)\|^{2}=0 \quad \text { a.s. }
$$

Proof. Choose a Lyapunov function

$$
V(k)=\widehat{\delta}^{T}(k h) \widehat{\delta}(k h) .
$$

It is not hard to show that both $H_{\sigma(k)}+H_{\sigma(k)}^{T}$ and $H_{\sigma(k)}^{T} H_{\sigma(k)}$ are positive definite for any $k \in \mathbb{Z}^{+}$since $\mathscr{G}_{\sigma(k)}$ is balanced and contains a spanning tree. It follows from (40) that

$$
\begin{aligned}
V(k+1) \leq & {\left[1-a(k) h \lambda_{\min }\left(H_{\sigma(k)}^{T}+H_{\sigma(k)}\right)\right.} \\
& \left.+a^{2}(k) h^{2} \lambda_{\max }\left(H_{\sigma(k)}^{T} H_{\sigma(k)}\right)\right] V(k) \\
+ & 2 a(k) h \widehat{\delta}^{T}(k h)\left(I-a(k) h H_{\sigma(k)}^{T}\right) U_{1}^{T} w(k h) \\
& +a^{2}(k) h^{2} w^{T}(k h) U_{1} U_{1}^{T} w(k h) .
\end{aligned}
$$

Denote $\lambda^{*}=\min _{k \geq 0}\left\{\lambda_{\min }\left(H_{\sigma(k)}^{T}+H_{\sigma(k)}\right)\right\}$ and $\lambda^{* *}=$ $\max _{k \geq 0}\left\{\lambda_{\max }\left(H_{\sigma(k)}^{T} H_{\sigma(k)}\right)\right\}$, which are well defined since $\mathscr{P}$ is a finite set. Thus,

$$
\begin{aligned}
V(k+1) \leq & {\left[1-a(k) h \lambda^{*}+a^{2}(k) h^{2} \lambda^{* *}\right] V(k) } \\
& +2 a(k) h \widehat{\delta}^{T}(k h)\left(I-a(k) h H_{\sigma(k)}^{T}\right) \\
& \times U_{1}^{T} w(k h) \\
& +a^{2}(k) h^{2} w^{T}(k h) U_{1} U_{1}^{T} w(k h) .
\end{aligned}
$$

The rest of the proof is similar to that in Theorem 8 and hence omitted. 


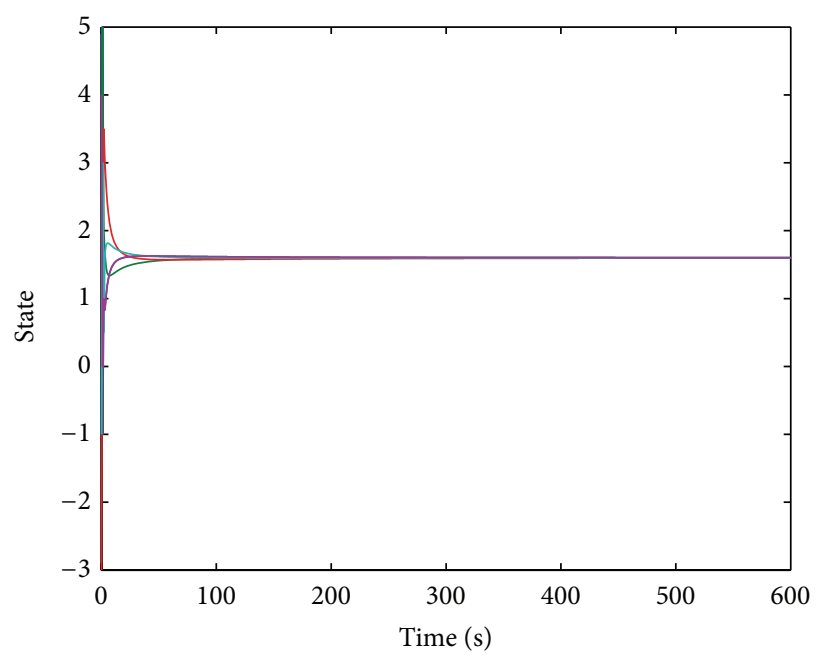

(a) $h=1$

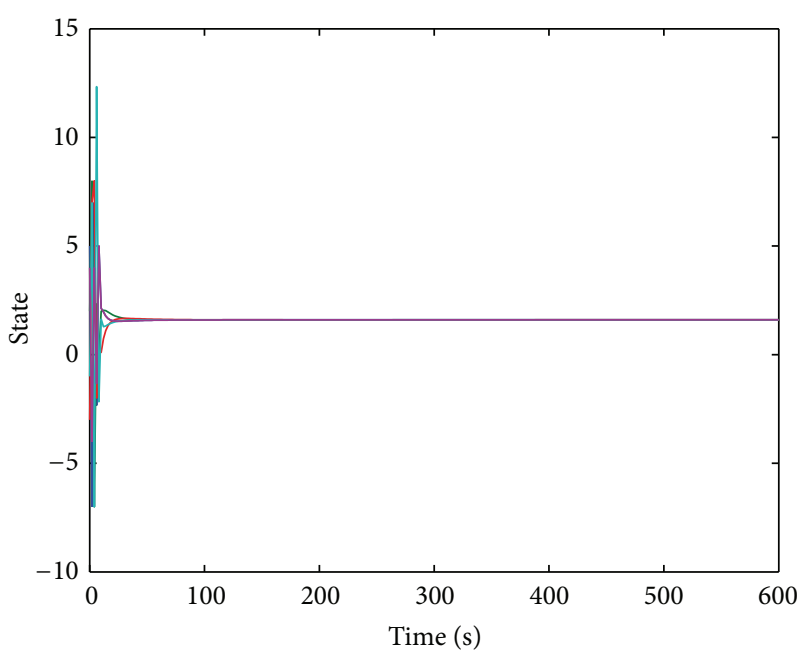

(b) $h=2$

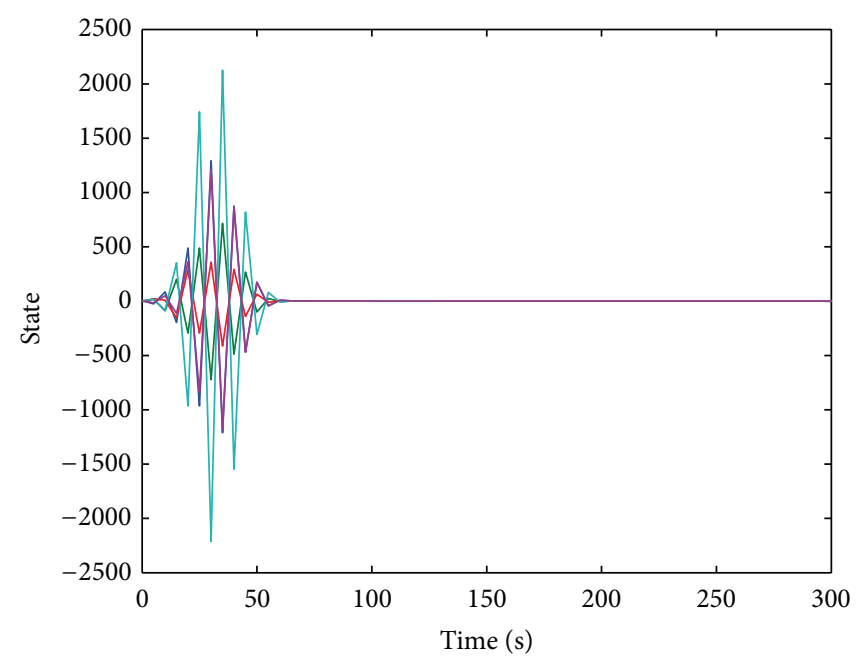

(c) $h=5$

FIgURE 2: State trajectories under different sampling periods in the case of noise-free and fixed topologies.

Theorem 14. Apply protocol (4) to system (2). Assume that (A1)-(A4) hold; then the $n$ agents reach almost sure consensus.

Proof. Note that

$$
\begin{aligned}
\|x(k h)-J x(k h)\|^{2} & =\left\|(I-J) U_{1} \widehat{\delta}(k h)\right\|^{2} \\
& \leq\left\|U_{1}^{T}(I-J) U_{1}\right\|\|\widehat{\delta}(k h)\|^{2},
\end{aligned}
$$

by (41).

The remaining of the proof is similar to that of Theorem 11 and hence omitted here.

Remark 15. Note that the condition in Theorem 14 that $\mathscr{G}_{\sigma(k)}$ is balanced is a sufficient condition. To weaken the condition on the network topology, it may require a new method. We leave this for further investigation.

\section{Simulations}

In this section, two examples are provided to illustrate the theoretical results. In the following two examples, the variance of the i.i.d zero mean Gaussian measurement noises is $\sigma^{2}=0.01$, and the step size $a(k)=1 /(k+1), k \geq 0$. It is clear that Assumptions (A1) and (A2) hold.

Example 16. Consider a multi-agent system consisting of five agents with the interaction topology shown in Figure 1. Note that $\mathscr{G}_{1}$ has a spanning tree. For simplicity, we assume that $\mathscr{G}_{1}$ has 0 - 1 weights. Let the initial value of $x(0)$ be taken randomly as $[5,2,-3,-1,4]^{T}$. Figures $2(\mathrm{a})-2$ (c) show that the five agents reach consensus under different sampling periods in the case of noise-free. Figures 3(a)-3(c) show that the states of the five agents converge to the same constant under different sampling periods with measurement noises. 


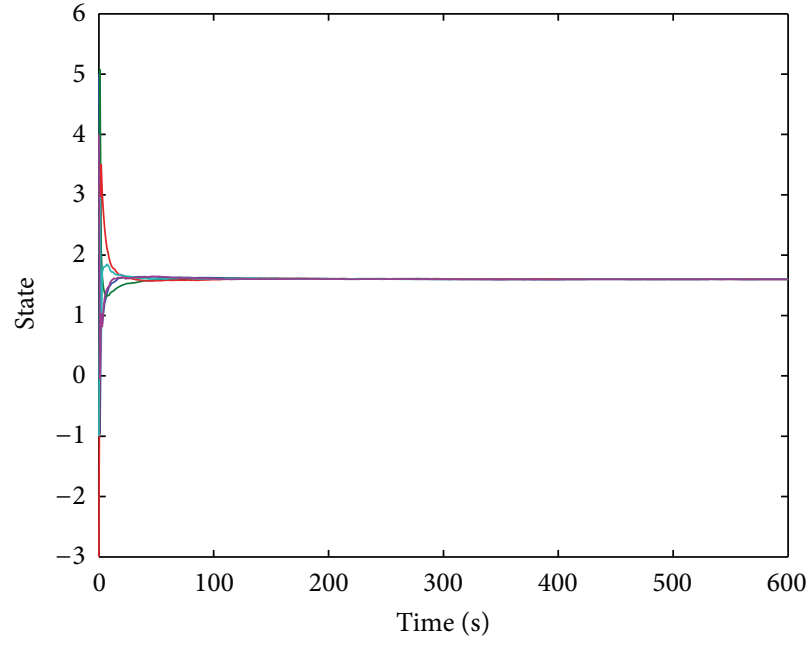

(a) $h=1$

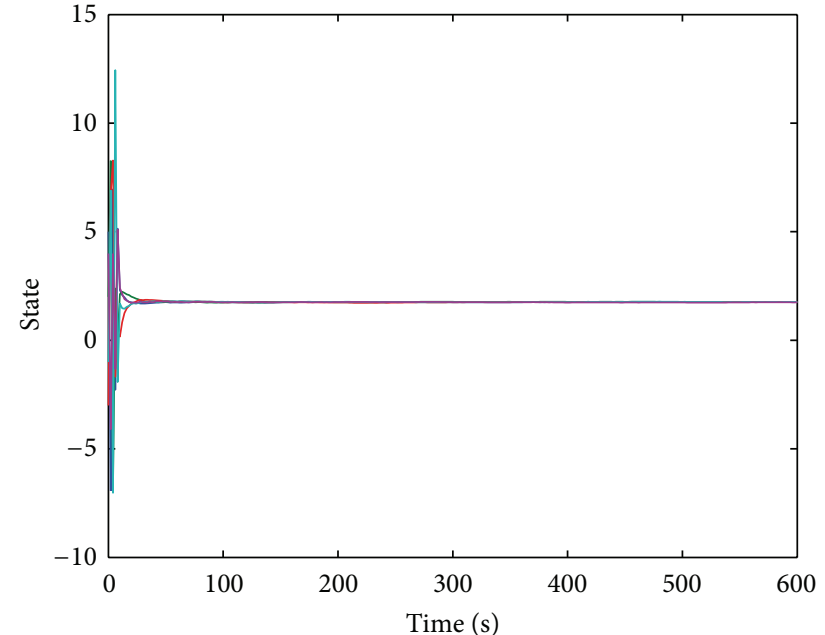

(b) $h=2$

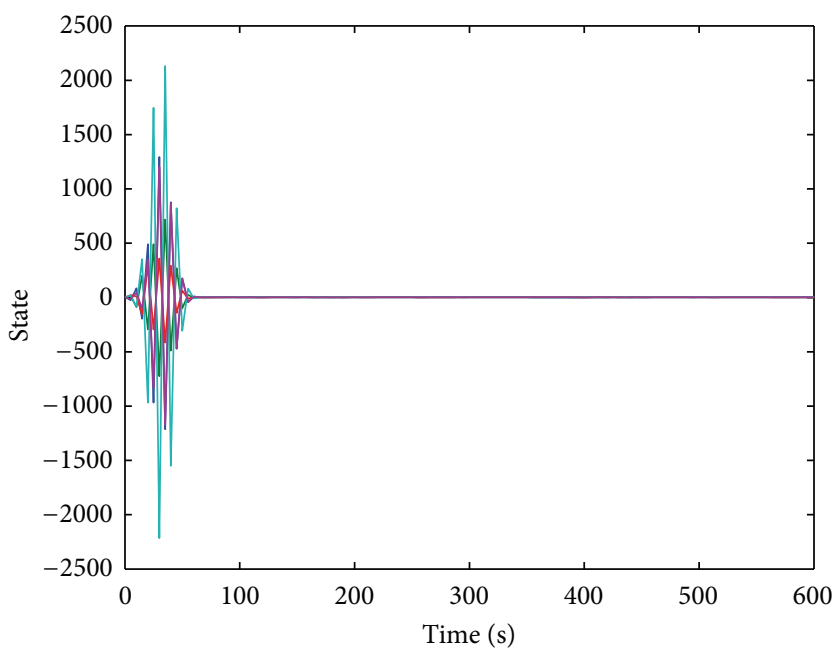

(c) $h=5$

FIGURE 3: State trajectories under different sampling periods with fixed topology and noisy measurements.

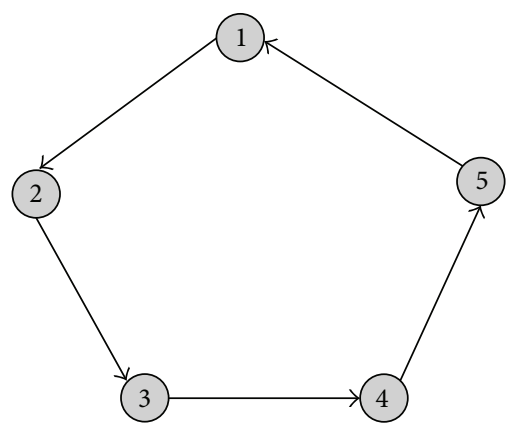

(a) $\mathscr{G}_{2}$

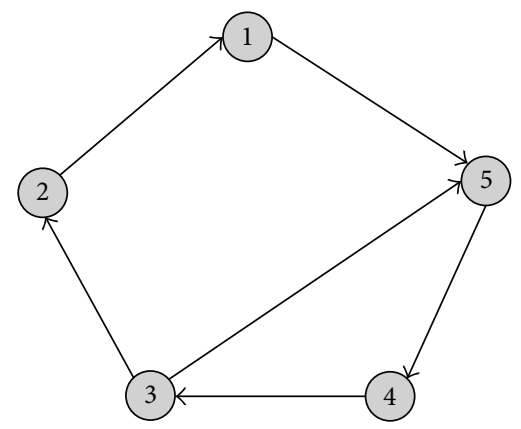

(b) $\mathscr{G}_{3}$

FigURE 4: Interaction topologies $\mathscr{G}_{2}$ and $\mathscr{G}_{3}$.

Example 17. Consider a multi-agent system consisting of five agents. The interaction topology is time varying of switching period $h$ between two graphs $\mathscr{G}_{i}(i=2,3)$ described as in Figure 4. For simplicity, we assume that $a_{34}=a_{45}=2$ in $\mathscr{G}_{3}$ and the weights of all the other edges in $\mathscr{G}_{2}$ and $\mathscr{G}_{3}$ are 1 . Note that both $\mathscr{G}_{2}$ and $\mathscr{G}_{3}$ are balanced and have a spanning tree. So, (A3) and (A4) are satisfied. It can be seen from Figures 5 (a) -5 (c) that the states of the five agents converge to the same 


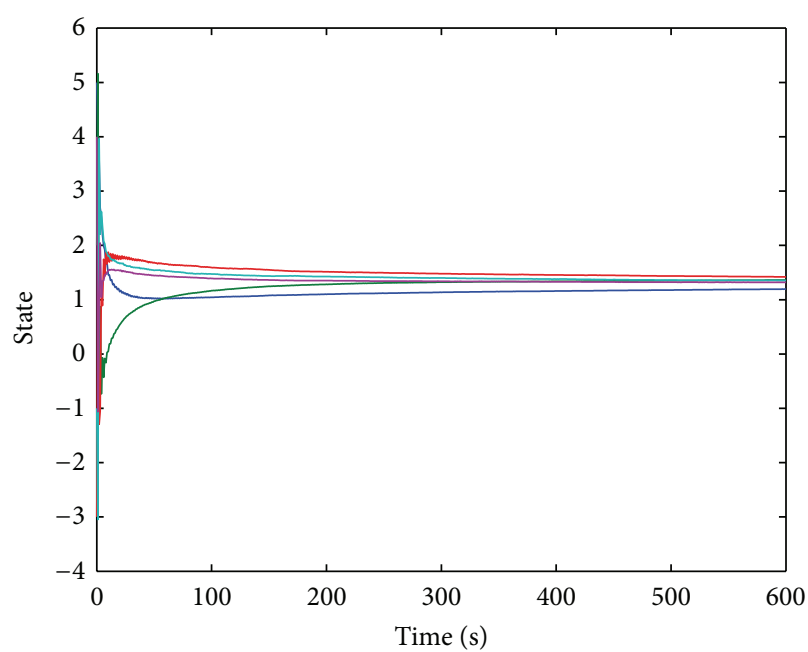

(a) $h=1$

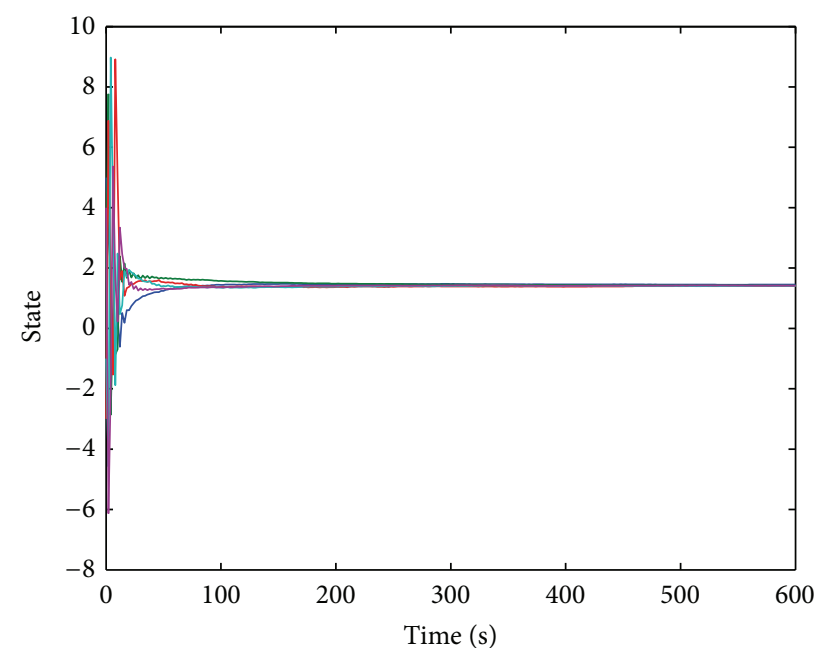

(b) $h=2$

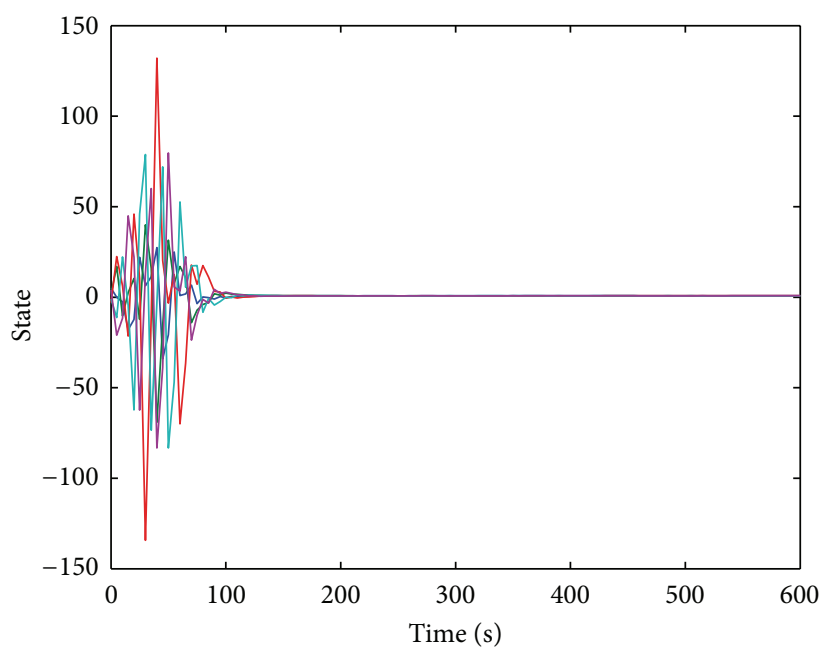

(c) $h=5$

FIGURE 5: State trajectories under different sampling periods with noisy measurements and time-varying topology.

constant under different sampling periods with measurement noises.

\section{Conclusion}

In this paper, a consensus problem for a multi-agent system with sampled information and noisy measurements is investigated. Both the case of fixed topology and time-varying topologies are taken into consideration. For the case of fixed topology, we prove that the agents reach almost sure strong consensus as long as the network topology contains a spanning tree. For the case of time-varying topologies, under the assumption that each interaction topology is balanced and contains a spanning tree, we show that the agents reach almost sure strong consensus. Different from the most existing results concerning sampled-data control of multiagent systems, it is shown that the convergence conditions are independent of the sampling period, which is due to the introduction of the step size.

\section{Acknowledgments}

This research is supported by Chinese Universities Specialized Research Fund for the Doctoral Program (20110185110020), Sichuan Province Science \& Technology Research Project (2012GZX0080).

\section{References}

[1] R. Olfati-Saber, J. A. Fax, and R. M. Murray, "Consensus and cooperation in networked multi-agent systems," Proceedings of the IEEE, vol. 95, no. 1, pp. 215-233, 2007.

[2] T. Vicsek, A. Czirók, E. Ben-Jacob, I. Cohen, and O. Shochet, "Novel type of phase transition in a system of self-driven particles," Physical Review Letters, vol. 75, no. 6, pp. 1226-1229, 1995.

[3] A. Jadbabaie, J. Lin, and A. S. Morse, "Coordination of groups of mobile autonomous agents using nearest neighbor rules," IEEE Transactions on Automatic Control, vol. 48, no. 6, pp. 988-1001, 2003. 
[4] R. Olfati-Saber and R. M. Murray, "Consensus problems in networks of agents with switching topology and time-delays," IEEE Transactions on Automatic Control, vol. 49, no. 9, pp. 15201533, 2004.

[5] L. Moreau, "Stability of multiagent systems with time-dependent communication links," IEEE Transactions on Automatic Control, vol. 50, no. 2, pp. 169-182, 2005.

[6] W. Ren and R. W. Beard, "Consensus seeking in multiagent systems under dynamically changing interaction topologies," IEEE Transactions on Automatic Control, vol. 50, no. 5, pp. 655-661, 2005.

[7] J. Hu and Y. Hong, "Leader-following coordination of multiagent systems with coupling time delays," Physica A, vol. 374, no. 2, pp. 853-863, 2007.

[8] Y. G. Sun and L. Wang, "Consensus of multi-agent systems in directed networks with uniform time-varying delays," IEEE Transactions on Automatic Control, vol. 54, no. 7, pp. 1607-1613, 2009.

[9] Y.-P. Tian and C.-L. Liu, "Consensus of multi-agent systems with diverse input and communication delays," IEEE Transactions on Automatic Control, vol. 53, no. 9, pp. 2122-2128, 2008.

[10] J. Cortés, "Finite-time convergent gradient flows with applications to network consensus," Automatica, vol. 42, no. 11, pp. 1993-2000, 2006.

[11] Q. Hui, W. M. Haddad, and S. P. Bhat, "Finite-time semistability and consensus for nonlinear dynamical networks," IEEE Transactions on Automatic Control, vol. 53, no. 8, pp. 1887-1900, 2008.

[12] L. Wang and F. Xiao, "Finite-time consensus problems for networks of dynamic agents," IEEE Transactions on Automatic Control, vol. 55, no. 4, pp. 950-955, 2010.

[13] M. Porfiri and D. J. Stilwell, "Consensus seeking over random weighted directed graphs," IEEE Transactions on Automatic Control, vol. 52, no. 9, pp. 1767-1773, 2007.

[14] A. Tahbaz-Salehi and A. Jadbabaie, "Consensus over ergodic stationary graph processes," IEEE Transactions on Automatic Control, vol. 55, no. 1, pp. 225-230, 2010.

[15] T. Hayakawa, T. Matsuzawa, and S. Hara, "Formation control of multi-agent systems with sampled information-relationship between information exchange structure and control performance," in Proceedings of the 45th IEEE Conference on Decision and Control (CDC '06), pp. 4333-4338, San Diego, Calif, USA, December 2006.

[16] Y. Cao, W. Ren, and Y. Li, "Distributed discrete-time coordinated tracking with a time-varying reference state and limited communication," Automatica, vol. 45, no. 5, pp. 1299-1305, 2009.

[17] Y. Cao and W. Ren, "Sampled-data discrete-time coordination algorithms for double-integrator dynamics under dynamic directed interaction," International Journal of Control, vol. 83, no. 3, pp. 506-515, 2010.

[18] Y. Gao and L. Wang, "Consensus of multiple dynamic agents with sampled information," IET Control Theory \& Applications, vol. 4, no. 6, pp. 945-956, 2010.

[19] M. Huang and J. H. Manton, "Coordination and consensus of networked agents with noisy measurements: stochastic algorithms and asymptotic behavior," SIAM Journal on Control and Optimization, vol. 48, no. 1, pp. 134-161, 2009.

[20] T. Li and J.-F. Zhang, "Consensus conditions of multi-agent systems with time-varying topologies and stochastic communication noises," IEEE Transactions on Automatic Control, vol. 55, no. 9, pp. 2043-2057, 2010.
[21] T. Li and J. Zhang, "Sampled-data based average consensus with measurement noises: convergence analysis and uncertainty principle," Science in China. Series F, vol. 52, no. 11, pp. 2089$2103,2009$.

[22] M. Huang and J. H. Manton, "Stochastic consensus seeking with noisy and directed inter-agent communication: fixed and randomly varying topologies," IEEE Transactions on Automatic Control, vol. 55, no. 1, pp. 235-241, 2010.

[23] H.-F. Chen, Stochastic Approximation and Its Applications, vol. 64 of Nonconvex Optimization and its Applications, Kluwer Academic Publishers, Dordrecht, The Netherlands, 2002.

[24] R. A. Horn and C. R. Johnson, Topics in Matrix Analysis, Cambridge University Press, New York, NY, USA, 1994.

[25] B. T. Polyak, Introduction to Optimization, Optimization Software, New York, NY, USA, 1987.

[26] T. C. Aysal, M. E. Yildiz, and A. Scaglione, "Broadcast gossip algorithms," in Proceedings of the IEEE Information Theory Workshop (ITW '08), pp. 343-347, Porto, Portugal, May 2008.

[27] P. Hall and C. C. Heyde, Martingale Limit Theory and Its Application, Academic Press, New York, NY, USA, 1980. 


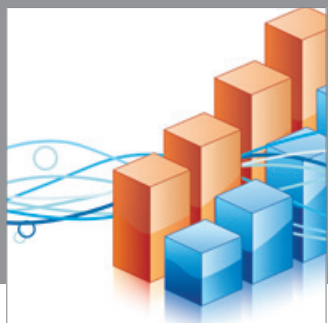

Advances in

Operations Research

mansans

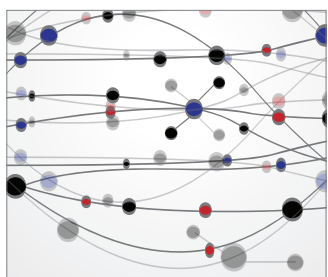

The Scientific World Journal
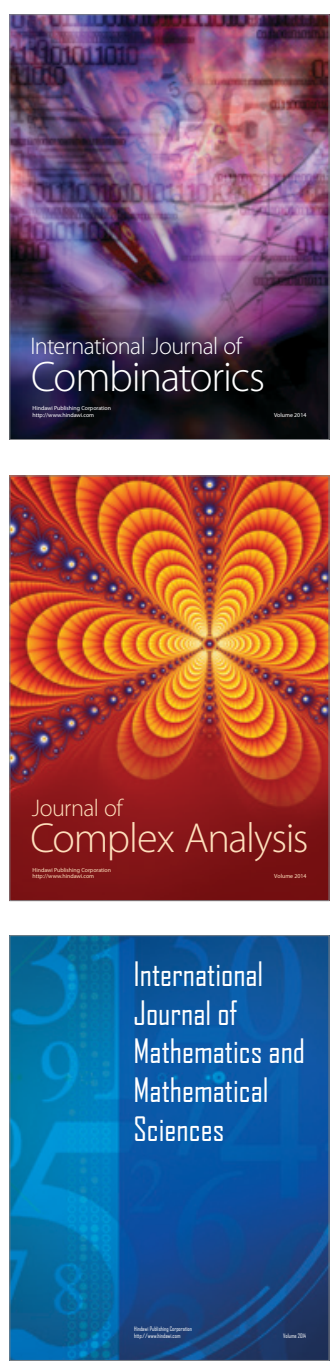
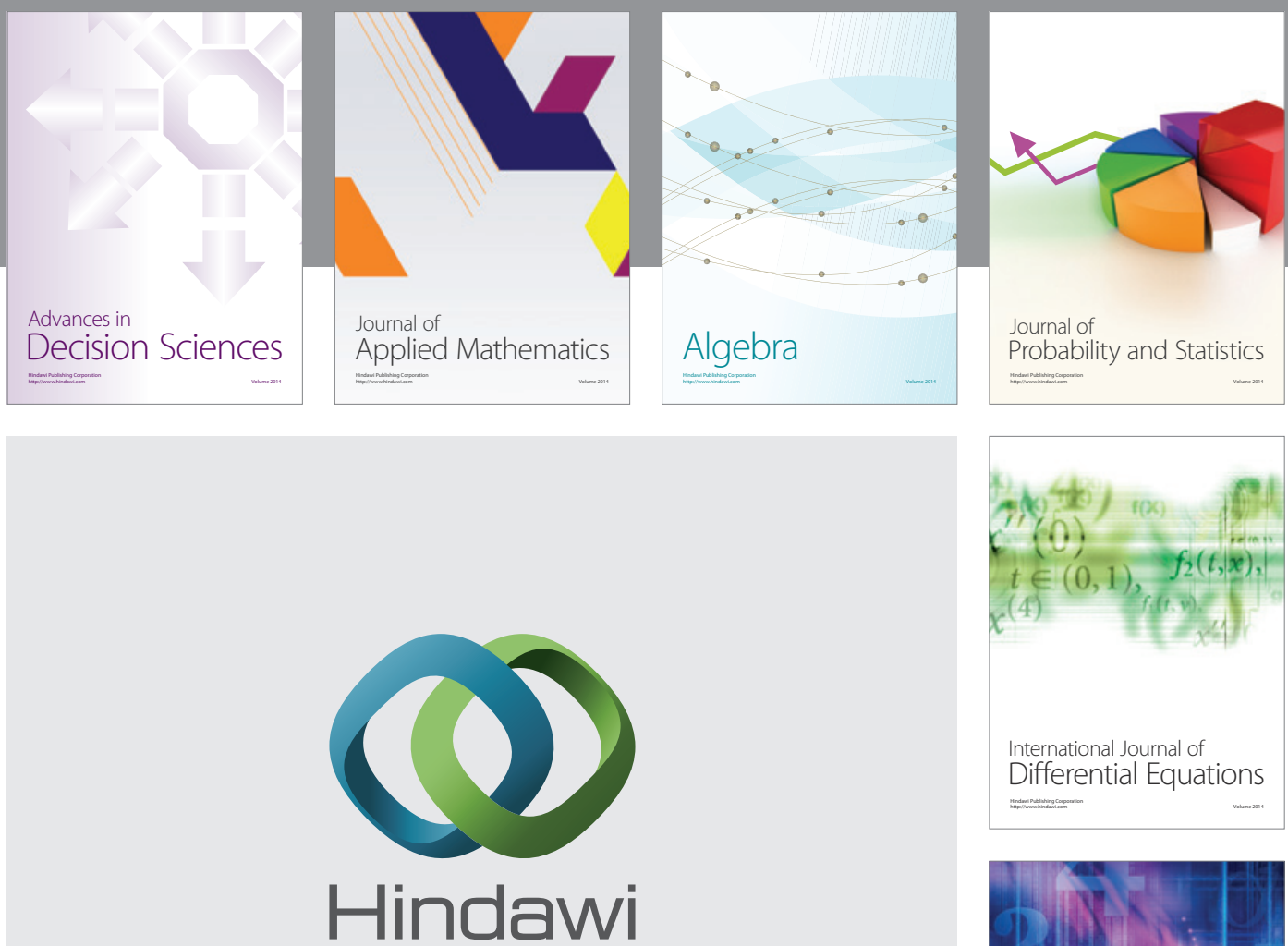

Submit your manuscripts at http://www.hindawi.com
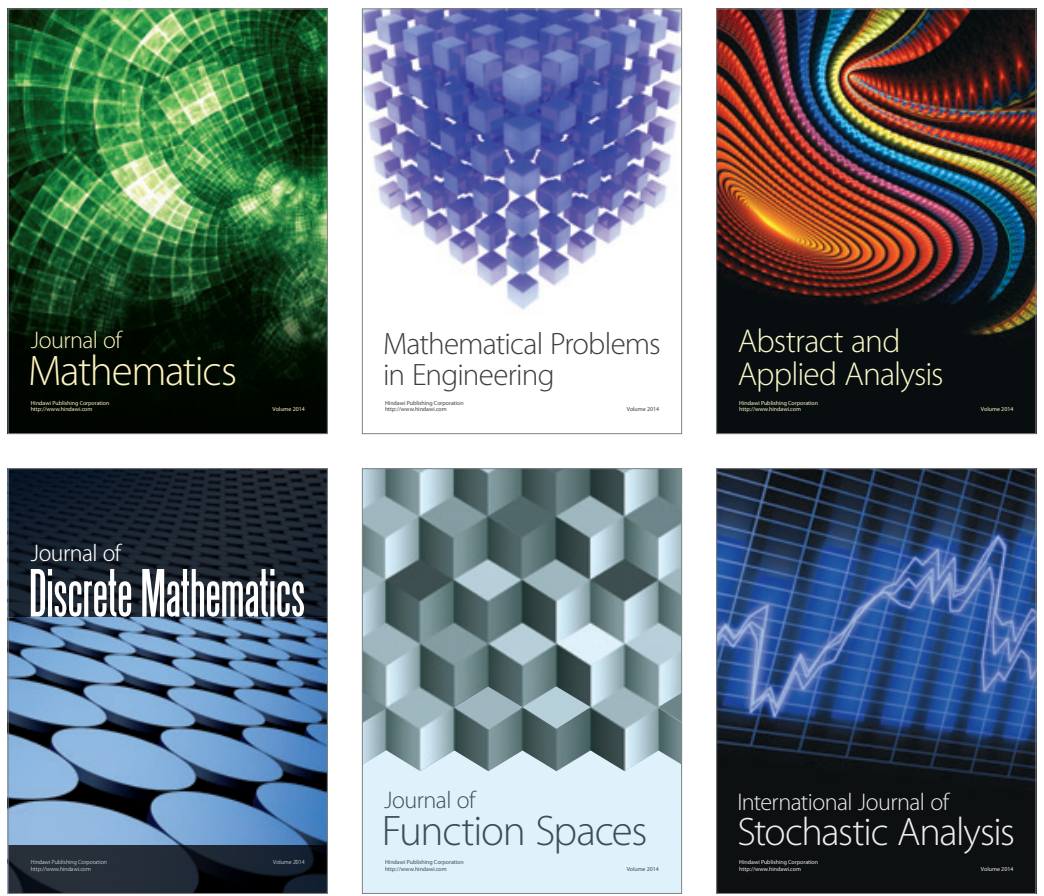

Journal of

Function Spaces

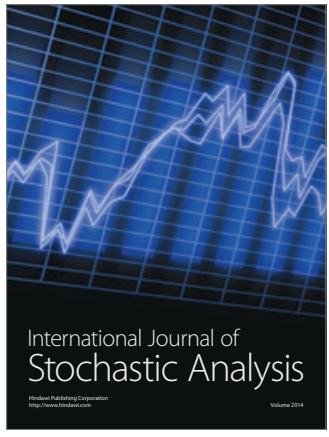

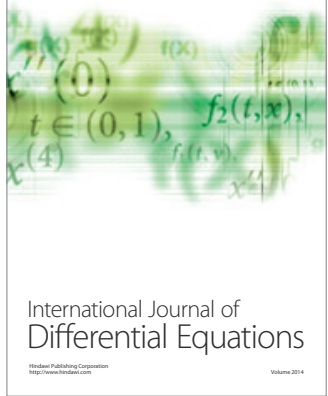
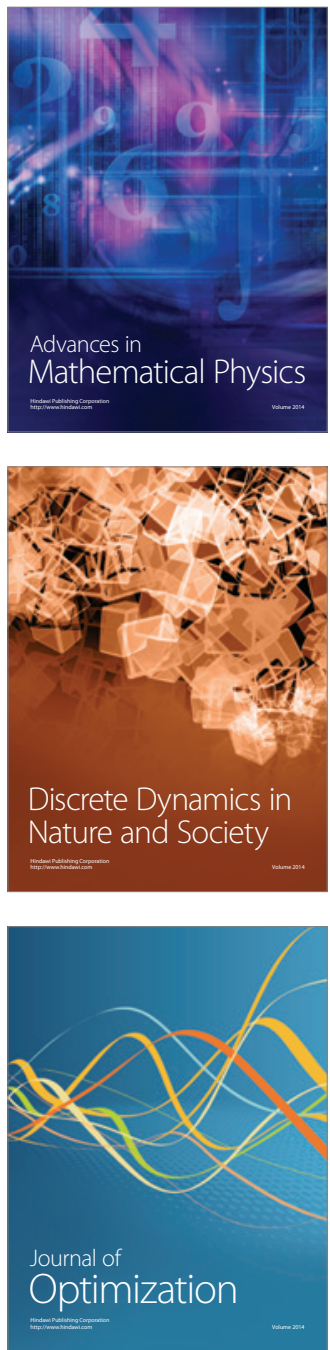